\title{
The finite element method for the mechanically based model of non-local continuum
}

\author{
M. Zingales*, $\dagger$, M. Di Paola and G. Inzerillo \\ Dipartimento di Ingegneria Strutturale, Aerospaziale e Geotecnica (DISAG), Università degli Studi di Palermo, \\ Viale delle Scienze Ed.8, I-90128, Palermo, Italy
}

\begin{abstract}
SUMMARY
In this paper the finite element method (FEM) for the mechanically based non-local elastic continuum model is proposed. In such a model each volume element of the domain is considered mutually interacting with the others, beside classical interactions involved by the Cauchy stress field, by means of central body forces that are monotonically decreasing with their inter-distance and proportional to the product of the interacting volume elements. The constitutive relations of the long-range interactions involve the product of the relative displacement of the centroids of volume elements by a proper, distance-decaying function, which accounts for the decrement of the long-range interactions as long as distance increases. In this study, the elastic problem involving long-range central interactions for isotropic elastic continuum will be solved with the aid of the FEM. The accuracy of the solution obtained with the proposed FEM code is compared with other solutions obtained with Galerkins' approximation as well as with finite difference method. Moreover, a parametric study regarding the effect of the material length scale in the mechanically based model and in the Kröner-Eringen non-local elasticity has been investigated for a plane elasticity problem. Copyright (C) 2011 John Wiley \& Sons, Ltd.
\end{abstract}

Received 14 October 2009; Revised 13 November 2010; Accepted 17 November 2010

KEY WORDS: non-local mechanics; long-range interactions; integro-differential equations; numerical methods; finite element analysis

\section{INTRODUCTION}

Classical continuum field theories, used in general context of elastic problems, yield useful solutions in good agreement with experimental evidences in all cases such that the resolution scale of the described problem is much larger than the internal dimension of the inner property of the material. Analysis of physical and engineering problems that does not fulfil this latter condition is nowadays faced in many contexts involving liquid crystals, biopolymers, shape memory alloys or micro/nanostructured materials. Indeed the presence of the material length scale is unavoidably loss in the continualization process leading to the field equations of continuum mechanics. The main observation about the discrete nature of matter led to the use of lattice dynamics theory in the analysis of engineering materials [1], but such an approach remains impracticable also for the most modern computer facilities, albeit it is questionable since it is not well established whether Newtonian mechanics apply at atomistic scale. As an alternative, some refinements of the theory of continuum mechanics have been proposed, since the beginning of the sixties of the last century, resorting to extensions of the theory of polar continuum [2], yielding the theory of micromorphic

\footnotetext{
${ }^{*}$ Correspondence to: M. Zingales, Dipartimento di Ingegneria Strutturale, Aerospaziale e Geotecnica (DISAG), Università degli Studi di Palermo, Viale delle Scienze Ed.8, I-90128, Palermo, Italy.

†E-mail: massimiliano.zingales@unipa.it 
continuum [3-6] or the quasi-continuum theories [7-10]. Indeed description of the inner state of the matter by means of micromorphic continuum theory yields the modern multiscale approach to computational mechanics [11-14] used very often in combination with Boundary Elements Methods [15]. On the other hand, quasi-continuum field theories have been proposed in several kind of physics and engineering problems since their first apparition at the beginning of seventies [8,16-19] and successfully applied in linear elasticity problems by closed-form solutions [20], finite element method (FEM) [21,22], as well as problems involving plasticity and damage [23-26] yielding smeared stress field in close vicinity of discontinuities of the considered domains. As an alternative, gradient methods accounting for the presence of an inner microstructure have been introduced in the nineties [27,28] and several applications of gradient theories have also been proposed in context of cracks propagations or plastic material behavior [24,29-32]. Beside the large amount of research efforts in the field of non-local mechanics that has been based on integral or gradient theories, at the beginning of the century a different non-local mechanics theory, dubbed peridynamic elasticity theory, has been introduced [33-37]. Within this theory long-range forces between different elements of an elastic body have been introduced to equilibrate external and inertial interactions. Such long-range forces result in integral terms that depend on the relative displacements of the interacting elements. In this way the requirement of differentiability of the response fields is no more necessary and a wide range of applications in the context of damage and fracture mechanics have been reported in the scientific literature [38].

Very recently an alternative approach in the field of non-local elasticity [39], dubbed mechanically based non-local elasticity has been proposed. This approach may be considered a fusion between the main concepts of the peridynamic theory, the fundamental of lattice dynamics as well as Eringen's integral model of elasticity [40]. This model is mainly a two-scale model in which, beside the classical local stress between adjacent volumes there is another kind of interaction between non-adjacent volumes accounting for the inner material microstructure. These latter are long-range central elastic bonds between non-adjacent volume elements. The long-range interactions are modeled as central body forces, monotonically decreasing with the inter-distance between interacting elements. The interactions have been assumed to depend on the relative displacements of the body elements and to be proportional to a proper, material dependent, distance-decaying function that is monotonically decreasing with the distance of interacting volumes. The proposed model of elastic continuum has proved to be thermodynamically consistent [41] as well as to fulfill all the basic theorems of linear elasticity theory [42]. The model, originally proposed in static setting for a simple 1D case has been extended to wave scattering [40] as well as to static analysis of general type of 3D elastic problems [43]. Remarkably, the mechanical boundary condition may be enforced without any difficulty since body forces do not affect the equilibrium of the Cauchy tetrahedron at the boundaries so that the traction-type boundary conditions involve, only, the Cauchy contact stresses.

The main governing equations of the theory, formulated in terms of displacement fields, are of integro-differential type and closed-form solutions could be obtained only for very specific functional class of decaying function and unbounded domains [44]; otherwise, finite differences or analytical, approximate strategies [45] must be used to provide approximate solutions. The main criticism to latter methods of solution of the governing equations of the mechanically based approach to non-local mechanics is related to the fine discretization grid necessary within finite difference methods (FDM) or to the difficulties in handling complex boundaries within approximate analytical methods.

The need for more reliable and easy-to-code numerical methods in handling non-local elastic problems with long-range interactions motivates the present study that extends the displacementbased version of the FEM to the analysis of elastic problem with long-range interactions.

The governing field equations of the elastic continuum with long-range central interactions have been formulated in a variational weak form and the solid domain has been discretized into non-local finite elements.

The element equations have been then obtained resorting to an approximation of the unknown field in the element domain with the use of shape functions. The resulting equilibrium matrix equations may be decomposed into the classical sparse, local stiffness matrix contribution due 
to the local Cauchy stress and a fully populated non-local stiffness matrix. Both matrices are symmetric, well conditioned and positive definite.

Some numerical applications involving 1D and 2D elastic problems are presented to show the capabilities of the proposed method of solution contrasting the FEM results with other results previously obtained via approximate analytical methods. The analysis reported in the paper shows that even for linear elements, accurate solution of the non-local elastic problem may be obtained. The paper is organized as follows: In Section 2 the main general concepts of the mechanically based approach to non-local mechanics is reported. In Section 3 the formulation of the non-local finite element model is provided on variational basis yielding the element equations for the general three-dimensional elasticity. Some numerical applications have been reported in Section 4. Section 5 has been devoted to a discussion of the connections and the differences between the mechanically based non-local elasticity, peridynamic non-local theory and Kröner-Eringen integral theory.

\section{THE MECHANICALLY BASED MODEL OF NON-LOCAL ELASTICITY}

In this section a brief outline of the mechanically based approach of the non-local model will be summarized. Let us consider a linear elastic solid embedded in a region $V$ of an Euclidean space and referred to the orthogonal reference system reported in Figure 1. Let us denote $S_{\mathrm{c}}$ the constrained part of the surface where the displacement are assigned and $S_{\mathrm{f}}$ the free part where the external tractions are assigned. Then $S=S_{\mathrm{f}} \cup S_{\mathrm{c}}$ is the total surface of the solid.

Let $\boldsymbol{u}(\boldsymbol{x})=\left\{u_{k}(\boldsymbol{x})\right\}$ be the displacement vector field at location $\boldsymbol{x}^{\mathrm{T}}=\left[x_{1}, x_{2}, x_{3}\right]$. In the following we denote $\overline{\boldsymbol{p}}_{n}$ the assigned tractions on $S_{f}$ and $\overline{\boldsymbol{u}}(\boldsymbol{x})$ the assigned displacements on $S_{\mathrm{c}}$, moreover let $\overline{\boldsymbol{b}}(\boldsymbol{x})=\left\{\bar{b}_{k}\right\}$ be the external body vector force field. The mechanically based model takes into account two kinds of interactions between different volumes: The contact stress (The classical Cauchy stress) and some non-local interactions between adjacent and non-adjacent volumes. The non-local interactions between solid volume elements are modeled as central body forces applied on volume element located in $\boldsymbol{x}$, denoted as $\mathrm{d} V(\boldsymbol{x})$, by each elementary volume elements located at position $\boldsymbol{\xi}$. Let $\boldsymbol{q}(\boldsymbol{x}, \boldsymbol{\xi})=\left\{q_{k}(\boldsymbol{x}, \boldsymbol{\xi})\right\}$ be the specific long-range interactions exerted at the location $\boldsymbol{x}$ by the volume element located in $\xi$. Since the non-local body forces are central, then the components of the specific long-range interactions vector field read:

$$
q_{k}(\boldsymbol{x}, \boldsymbol{\xi})=|\boldsymbol{q}(\boldsymbol{x}, \boldsymbol{\xi})| r_{k}(\boldsymbol{x}, \boldsymbol{\xi}), \quad k=1,2,3
$$

where $|\boldsymbol{q}(\boldsymbol{x}, \boldsymbol{\xi})|=\left(q_{k}(\boldsymbol{x}, \boldsymbol{\xi}) q_{k}(\boldsymbol{x}, \boldsymbol{\xi})\right)^{1 / 2}$ is the norm of long-range interactions (convention of repeated symbols is adopted), and $r_{k}(\boldsymbol{x}, \boldsymbol{\xi})$ the $k$ th component of the unitary vector associated with the direction $\boldsymbol{x}-\boldsymbol{\xi}$, and then it reads

$$
r_{k}(\boldsymbol{x}, \boldsymbol{\xi})=\frac{\left(x_{k}-\xi_{k}\right)}{\left[\left(x_{i}-\xi_{i}\right)^{2}\right]^{1 / 2}}
$$

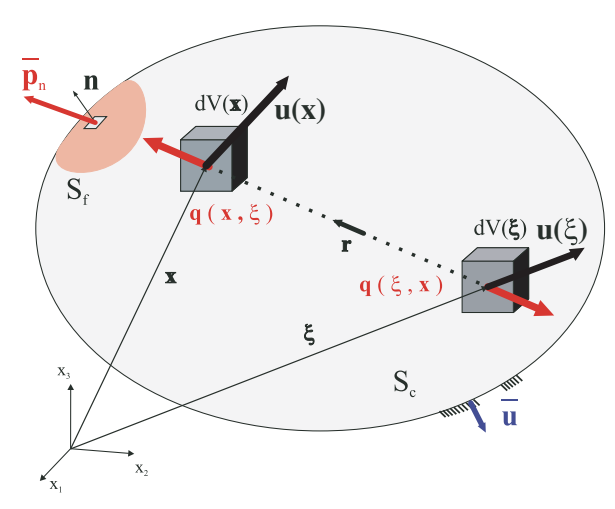

Figure 1. Volume elements in 3D solid. 
Vector $\boldsymbol{q}(\boldsymbol{x}, \boldsymbol{\xi})$ is modeled as:

$$
q(x, \xi)=G(x, \xi) \cdot \eta(x, \xi)
$$

where $[\cdot] \cdot[\cdot]$ is the tensor contraction operator, $\boldsymbol{\eta}(\boldsymbol{x}, \boldsymbol{\xi})=\left\{\eta_{k}(\boldsymbol{x}, \boldsymbol{\xi})\right\}$ is the relative displacements field represented by $\boldsymbol{\eta}(\boldsymbol{x}, \boldsymbol{\xi})=(\boldsymbol{u}(\boldsymbol{x})-\boldsymbol{u}(\boldsymbol{\xi}))$ and the directional double symmetric tensor $\boldsymbol{G}(\boldsymbol{x}, \boldsymbol{\xi})=$ $\left\{g_{i k}(\boldsymbol{x}, \boldsymbol{\xi})\right\}$ gathers, under the assumption of isotropic solid, elements in the form:

$$
g_{i k}(\boldsymbol{x}, \boldsymbol{\xi})=g(\boldsymbol{x}, \boldsymbol{\xi}) J_{i k}(\boldsymbol{x}, \boldsymbol{\xi})
$$

being $\boldsymbol{J}(\boldsymbol{x}, \boldsymbol{\xi})=\left\{J_{i k}(\boldsymbol{x}, \boldsymbol{\xi})\right\}$ the Jacobi tensor defined as: $J_{i k}(\boldsymbol{x}, \boldsymbol{\xi})=r_{i}(\boldsymbol{x}, \boldsymbol{\xi}) r_{k}(\boldsymbol{x}, \boldsymbol{\xi})$ (see [41]). The scalar-valued function $g(\boldsymbol{x}, \boldsymbol{\xi})$ is a distance-decaying function that accounts for the consideration that all long-range interactions decay with the interdistance $|x-\xi|$ and it will be assumed in the form: $g(\boldsymbol{x}, \boldsymbol{\xi})=g(|\boldsymbol{x}-\boldsymbol{\xi}|)$.

The introduction of the long-range interactions among non-adjacent volume elements involves additional terms in the field equilibrium equation. The resultant non-local elastic equilibrium problem is governed by the system of differential equations:

$$
\begin{aligned}
\operatorname{div}\left[\boldsymbol{\sigma}^{(1)}(\boldsymbol{x})\right] & =-\overline{\boldsymbol{b}}(\boldsymbol{x})-\boldsymbol{f}(\boldsymbol{x}), \quad \boldsymbol{x} \in V \\
\boldsymbol{\varepsilon}(\boldsymbol{x}) & =\nabla^{S}[\boldsymbol{u}(\boldsymbol{x})], \quad \boldsymbol{x} \in V \\
\boldsymbol{\sigma}^{(\mathrm{l})}(\boldsymbol{x}) & =\boldsymbol{D}: \boldsymbol{\varepsilon}, \quad \boldsymbol{x} \in V
\end{aligned}
$$

where $\operatorname{div}[\cdot]$ is the divergence operator $\left(\operatorname{div}\left[\boldsymbol{\sigma}^{(1)}(\boldsymbol{x})\right]=\left\{\sigma_{i k, k}(\boldsymbol{x})\right\}\right)$ and $[\cdot]_{, i}$ means differentiation with respect to the field variable $x_{i}$. We denoted $\boldsymbol{\sigma}^{(\mathrm{l})}(\boldsymbol{x})=\left\{\sigma_{i k}(\boldsymbol{x})\right\}$ the double and symmetric Cauchy tensor field, $\boldsymbol{\varepsilon}(\boldsymbol{x})=\left\{\varepsilon_{i k}(\boldsymbol{x})\right\}$ the, double and symmetric, strain tensor field that related to the symmetric component of the gradient operator as: $\nabla^{s}[\boldsymbol{u}(\boldsymbol{x})]=\left\{1 / 2\left(u_{i, k}(\boldsymbol{x})+u_{k, i}(\boldsymbol{x})\right)\right\}$. In the constitutive relation in Equation $(5 \mathrm{c}) \boldsymbol{D}=\left\{D_{i k m l}\right\}$ is the symmetric, fourth-order tensor of the elastic constants that, under the assumption of hyperelastic and isotropic material, is expressed in terms of Lamé constants $\lambda$ and $\mu$ as

$$
D_{i k m l}=\lambda \delta_{i k} \delta_{m l}+\mu\left(\delta_{i m} \delta_{k l}+\delta_{i l} \delta_{k m}\right)
$$

where $\delta_{i k}$ is the Kronecker delta. The Lamé constants are related to Young's modulus $E$ and to the Poisson ratio $v$ by the well-known expression:

$$
\lambda=\frac{E v}{(1+v)(1-2 v)} \quad \mu=\frac{E}{2(1+v)}
$$

The extra term in the equilibrium equation (5a), $\boldsymbol{f}(\boldsymbol{x})=\left\{f_{k}(\boldsymbol{x})\right\}$ is the resultant of the internal long-range interactions applied on volume $\mathrm{d} V(\boldsymbol{x})$ by all the surrounding non-adjacent volume elements of the body that is represented as

$$
\boldsymbol{f}(\boldsymbol{x})=\int_{V} \boldsymbol{q}(\boldsymbol{x}, \boldsymbol{\xi}) \mathrm{d} V(\boldsymbol{\xi})=\int_{V} \boldsymbol{G}(\boldsymbol{x}, \boldsymbol{\xi}) \cdot \boldsymbol{\eta}(\boldsymbol{x}, \boldsymbol{\xi}) \mathrm{d} V(\boldsymbol{\xi})
$$

with each component as:

$$
f_{k}(\boldsymbol{x})=\int_{V} q_{k}(\boldsymbol{x}, \boldsymbol{\xi}) \mathrm{d} V(\boldsymbol{\xi})=\int_{V} g_{k i}(\boldsymbol{x}, \boldsymbol{\xi}) \eta_{i}(\boldsymbol{x}, \boldsymbol{\xi}) \mathrm{d} V(\boldsymbol{\xi})
$$

The governing equations of the elastic problem formulated in Equations (5)-(9) may be cast, in Navier form involving only kinematic variables as:

$$
\mu \nabla^{2} u_{k}(\boldsymbol{x})+(\lambda+\mu) \theta_{1, k}(\boldsymbol{x})+\int_{V} g_{k i}(\boldsymbol{x}, \boldsymbol{\xi}) \eta_{i}(\boldsymbol{x}, \boldsymbol{\xi}) \mathrm{d} V(\boldsymbol{\xi})+\bar{b}_{k}=0
$$


where $\theta_{1, k}(\boldsymbol{x})=u_{i, i k}$ is the gradient of the first invariant of the strain tensor $\boldsymbol{\varepsilon}$ and $\nabla^{2}[\cdot]=[\cdot]_{, i i}$ is the Laplacian operator. Static and kinematic boundary conditions associated with the field governing equations in Equation (10) reads [43]

$$
\begin{aligned}
\boldsymbol{u}(\boldsymbol{x}) & =\overline{\boldsymbol{u}}(\boldsymbol{x}) \quad \boldsymbol{x} \in S_{\mathrm{c}} \\
\boldsymbol{\sigma}^{(\mathrm{l})}(\boldsymbol{x}) \cdot \boldsymbol{n} & =\overline{\boldsymbol{p}}_{n} \quad \boldsymbol{x} \in S_{\mathrm{f}}
\end{aligned}
$$

the governing equations of the static equilibrium problem in Equations (10) and (11) may also be obtained as the Euler-Lagrange equations and associated natural boundary of the total potential energy of the elastic solid with long-range interactions. It is to be stressed that the mechanically boundary conditions in the mechanically based theory simply remain the classical ones since the non-local forces are body forces and then they do not affect the equilibrium equations of the Cauchy tetrahedron at the boundaries. More details on this crucial point of the theory may be found in [43].

It has been shown in previous papers (see e.g. [43]) that the mechanically based non-local elasticity theory may be contrasted, efficiently, with the Kröner-Eringen non-local elasticity theory in the 1D case either for unbounded and bounded domains. Moreover, in 1D analysis an exhaustive discussion between the mechanically based model of non-local elasticity, the Kröner-Eringen nonlocal elasticity and the peridynamic theory has been reported in [40]. Under the assumption of vanishing contact stress, the mechanically based model of non-local elasticity is exactly coalescing with the peridynamic theory in the $1 \mathrm{D}$ case. The aforementioned observations lead to the conclusion that, in 1D case, the mechanically based model of non-local elasticity may be considered a hybrid model between different non-local theories in unbounded domains. In bounded domains, instead, the mechanical boundary conditions may be imposed in terms of the local stress as the applied surface tractions at the boundaries as already discussed. This cannot be done with lattice theory or the peridynamic theory because, at the boundaries of the elastic domain, the external pressure, which is a contact interaction, cannot be equilibrated by volume body forces.

In a generic 3D problem, a direct comparison of the expressions of the long-range forces involved by the peridynamic theory of non-local elasticity [33] with the interactions involved by the mechanically based non-local elasticity shows that they coincide, as far as the assumption of unstressed reference state is postulated in the peridynamic model (see Equation (61) in [33]).

On one hand this consideration may lead to consider the mechanically based model of non-local elasticity as a particular case of peridynamic theory, in the unstressed reference state, involving the additional contribution of the Cauchy contact stress to account for the traction-type boundary conditions. On the other hand, it exhibits a main difference between those two theories in the presence of a distributed set of long-range forces in the reference state of the solid, repulsive or attractive for some values of the interaction distances that, in the peridynamic theory, do not contain any Jacoby directional tensor [33]. As in fact, it may be observed from straightforward manipulation of Equations (53), (61) in [33] that in the presence of internal forces, non-central terms are involved in the peridynamic models. A different scenario is encountered in the mechanically based non-local elasticity since the existence of an internal body force field in the unstressed state must be accounted introducing an initial body force field represented by a Jacoby directional tensor. In fact the mechanically based model of non-local elasticity is based on the assumption that all the long-range interactions are always modeled by central-type long-range forces as in the complex network of springs of lattice dynamics. In passing we remark that the presence of long-range interactions beside the classical Cauchy stress as in the mechanically based model of non-local elasticity corresponds to a multiscale mechanical model. In fact local Cauchy stress is experienced at macroscopic level, whereas the long-range interactions appear as we consider a refined model of the solid body (i.e. a mesoscopic picture of the solid) useful to describe fracture mechanics or voids nucleation in the material structure.

Summing up the mechanically based model of non-local elasticity may be considered formally similar to the peridynamic theory but it is de facto a different model since it has been built upon the mechanical equivalence of a complex network of springs. This model corresponds, always to a central-type model of long-range interactions also in the presence of internal forces in the 
reference configuration. The model, which is intrinsically a multiscale description of the solid body behavior, may be enriched of additional contributions as the presence of long-range moments describing the behavior of polar materials. This is, anyway, beyond the scope of the paper and it will be considered in a separate study.

The variational form of the elastic equilibrium problem will be used in the next section to formulate the finite element equations of the elastic problem with long-range central interactions.

\section{DISPLACEMENT-BASED FINITE ELEMENTS IN THE PRESENCE OF LONG-RANGE CENTRAL INTERACTIONS}

The elastic problem reported in the previous section may be cast in terms of the total elastic potential energy. This is a convenient formulation to provide the basic element equations of the FEM. Similar considerations may be provided resorting to a weak form of the governing integrodifferential equation reported in Equations (10) and (11) (see [42,45]). The total potential energy $\Pi(\boldsymbol{u}, \boldsymbol{\varepsilon}, \boldsymbol{\eta})$ of the elastic equilibrium problem is defined as $\Pi(\boldsymbol{u}, \boldsymbol{\varepsilon}, \boldsymbol{\eta})=\Phi(\boldsymbol{\varepsilon}, \boldsymbol{\eta})+P(\boldsymbol{u})$ where the potential energy of the external, conservative loads $P(\boldsymbol{u})$ is represented by

$$
P(\boldsymbol{u})=\int_{V} P^{(r)}(\boldsymbol{u}, \boldsymbol{x}) \mathrm{d} V(\boldsymbol{x})+\int_{S_{\mathrm{f}}} P^{(s)}(\boldsymbol{u}, \boldsymbol{x}) \mathrm{d} S_{\mathrm{f}}(\boldsymbol{x})
$$

where $P^{(r)}(\boldsymbol{u}, \boldsymbol{x})$ and $P^{(s)}(\boldsymbol{u}, \boldsymbol{x})$ are given by

$$
P^{(r)}(\boldsymbol{u}, \boldsymbol{x})=-\overline{\boldsymbol{b}}(\boldsymbol{x}) \cdot \boldsymbol{u}(\boldsymbol{x}), \quad P^{(s)}(\boldsymbol{u}, \boldsymbol{x})=-\overline{\boldsymbol{t}}_{n}(\boldsymbol{x}) \cdot \boldsymbol{u}(\boldsymbol{x})
$$

and $\Phi(\boldsymbol{\varepsilon}, \boldsymbol{\eta})$ is the elastic potential energy of the solid that is provided in the form $[42,43]$ :

$$
\Phi(\boldsymbol{\varepsilon}, \boldsymbol{\eta})=\frac{1}{2} \int_{V} \boldsymbol{\varepsilon}(\boldsymbol{x}): \boldsymbol{D}: \boldsymbol{\varepsilon}(\boldsymbol{x}) \mathrm{d} V(\boldsymbol{x})+\frac{1}{4} \int_{V} \int_{V} \boldsymbol{\eta}(\boldsymbol{x}, \boldsymbol{\xi}) \cdot \boldsymbol{G}(\boldsymbol{x}, \boldsymbol{\xi}) \cdot \boldsymbol{\eta}(\boldsymbol{x}, \boldsymbol{\xi}) \mathrm{d} V(\boldsymbol{\xi}) \mathrm{d} V(\boldsymbol{x})
$$

so that the total potential energy of the elastic problem giving the governing equations already shown in Section 2 reads:

$$
\begin{aligned}
\Pi(\boldsymbol{u}, \boldsymbol{\varepsilon}, \boldsymbol{\eta})= & \frac{1}{2} \int_{V} \boldsymbol{\varepsilon}(\boldsymbol{x}): \boldsymbol{D}: \boldsymbol{\varepsilon}(\boldsymbol{x}) \mathrm{d} V(\boldsymbol{x})+\frac{1}{4} \int_{V} \int_{V} \boldsymbol{\eta}(\boldsymbol{x}, \boldsymbol{\xi}) \cdot \boldsymbol{G}(\boldsymbol{x}, \boldsymbol{\xi}) \cdot \boldsymbol{\eta}(\boldsymbol{x}, \boldsymbol{\xi}) \mathrm{d} V(\boldsymbol{\xi}) \mathrm{d} V(\boldsymbol{x}) \\
& -\int_{V} P^{(r)}(\boldsymbol{u}, \boldsymbol{x}) \mathrm{d} V(\boldsymbol{x})-\int_{S_{\mathrm{f}}} P^{(s)}(\boldsymbol{u}, \boldsymbol{x}) \mathrm{d} S_{\mathrm{f}}(\boldsymbol{x})
\end{aligned}
$$

It has been proved in a previous paper [45] that the solution of the elastic problem corresponds to a minimum of Equation (15). Analytical solutions of the 3D elastic problem with long-range forces non-local interactions are not available and only some numerical approximate solution based of finite differences have been previously exploited [43].

In the FEM solution we basically introduce a proper discrete mesh subdividing the volume $V$ of the solid into disjoint volume elements $V_{i}(i, j=1,2, \ldots, n)$ with common vertices shared by adjacent volumes defined as mesh nodes. The discrete mesh is such that $V=V_{1} \cup V_{2} \cup \cdots \cup V_{n}$. So that the following relation:

$$
\Pi(\boldsymbol{u}, \boldsymbol{\varepsilon}, \boldsymbol{\eta})=\sum_{i=1}^{n} \Pi_{i}(\boldsymbol{u}, \boldsymbol{\varepsilon}, \boldsymbol{\eta})=\sum_{i=1}^{n}\left(\Phi_{i}(\boldsymbol{\varepsilon}, \boldsymbol{\eta})+P_{i}^{(r)}(\boldsymbol{u})+P_{i}^{(s)}(\boldsymbol{u})\right)
$$

holds true. The approximation involved in the finite element scheme is related to the interpolation of the displacement field within the volume element $V_{i}(\boldsymbol{x})$, denoted $\boldsymbol{u}_{i}(\boldsymbol{x})$ and represented in the form:

$$
\boldsymbol{u}_{i}(\boldsymbol{x})=\boldsymbol{N}_{i}(\boldsymbol{x}) \boldsymbol{d}_{i}, \quad i=1,2, \ldots, n
$$


where $N_{i}$ is the matrix collecting the interpolation functions and $\boldsymbol{d}_{i}$ is the vector of the unknown nodal displacement. The element equations of the proposed model of non-local elasticity may be obtained introducing Equation (17) into the generic contribution in Equation (16), yielding:

$$
\Pi_{i}\left(\boldsymbol{u}_{i}, \boldsymbol{\varepsilon}_{i}, \boldsymbol{\eta}\right) \cong \Phi_{i}\left(\boldsymbol{\varepsilon}_{i}, \boldsymbol{\eta}\right)+\int_{V_{i}} P_{i}^{(r)}\left(\boldsymbol{u}_{i}, \boldsymbol{x}\right) \mathrm{d} V_{i}(\boldsymbol{x})+\int_{S_{i}} P_{i}^{(s)}\left(\boldsymbol{u}_{i}, \boldsymbol{x}\right) \mathrm{d} S_{i}(\boldsymbol{x})
$$

where the elastic potential energy of the $i$ th volume element is expressed in the form:

$$
\begin{aligned}
\Phi_{i}\left(\boldsymbol{\varepsilon}_{i}, \boldsymbol{\eta}\right)= & \frac{1}{2} \boldsymbol{d}_{i}^{\mathrm{T}} \int_{V_{i}} \boldsymbol{B}_{i}(\boldsymbol{x})^{\mathrm{T}} \overline{\boldsymbol{D}} \boldsymbol{B}_{i}(\boldsymbol{x}) \mathrm{d} V_{i}(\boldsymbol{x}) \boldsymbol{d}_{i} \\
& +\frac{1}{4} \sum_{l=1}^{n} \int_{V} \int_{V_{i}}[\boldsymbol{\eta}(\boldsymbol{x}, \boldsymbol{\xi})]^{\mathrm{T}} \boldsymbol{G}(\boldsymbol{x}, \boldsymbol{\xi}) \boldsymbol{\eta}(\boldsymbol{x}, \boldsymbol{\xi}) \mathrm{d} V_{i}(\boldsymbol{x}) \mathrm{d} V(\boldsymbol{\xi})
\end{aligned}
$$

where we denoted $\overline{\boldsymbol{D}}$ the $6 \times 6$ compliance matrix of the material, listing elements $\bar{D}_{i j}=$ $-\lambda \delta_{i j} /[2 \mu(3 \lambda+2 \mu)]+1 /(2 \mu)$, corresponding to the fourth-order tensor $\boldsymbol{D}$ as major and minor symmetries have been accounted for, and

$$
\begin{aligned}
B_{i}(x) & =\nabla^{s}\left[N_{i}(x)\right] \\
\eta(x, \xi) & =u(\xi)-u(x)=N(\xi) d_{j}-N_{i}(x) d_{i}
\end{aligned}
$$

The main consideration that may be withdrawn from the observation of Equation (19) is related to the sum of $n$ double integral at the right-hand side of Equation (19) that is extended to each couple of $\mathrm{d} V$ and $\mathrm{d} V_{i} j=1,2, \ldots, n$ in the solid body domain. The presence of this additional term is due to the long-range interactions as it represents the amount of elastic energy stored in the long-range bonds. Moreover, it may be observed that the elastic potential of element $V_{i}$ do involve displacement field in the whole body due to the sum in Equation (19). To this aim let us define the $N$-dimensional nodal displacement vector of the whole discretized structure as $\boldsymbol{d}^{\mathrm{T}}=\left[d_{1} d_{2} \ldots d_{N}\right]$ gathering the nodal displacements of all the nodes of the solid body elements so that the nodal displacement $\boldsymbol{d}_{i}$ may be obtained by means of the boolean, connectivity matrix $\boldsymbol{C}_{i}$ of the $i$ th element as:

$$
\boldsymbol{d}_{i}=\boldsymbol{C}_{i} \boldsymbol{d}, \quad i=1,2, \ldots, n
$$

Substitution of Equations (20b) and (21) into Equation (19) yields the compact expression:

$$
\Phi_{i}\left(\boldsymbol{\varepsilon}_{i}, \boldsymbol{\eta}\right)=\frac{1}{2}\left(\boldsymbol{d}^{\mathrm{T}}\left(\boldsymbol{K}_{i}^{(\mathrm{l})}+\boldsymbol{K}_{i}^{(\mathrm{nl})}\right) \boldsymbol{d}\right)
$$

where we denoted $\boldsymbol{K}_{i}^{(\mathrm{l})}$ the classical local stiffness matrix accounting for contact stress of the $i$ th volume element and $\boldsymbol{K}_{i}^{(\mathrm{nl})}$ is the non-local stiffness matrix accounting for the long-range interactions. These two stiffness matrices may be expressed in the form:

$$
\begin{aligned}
\boldsymbol{K}_{i}^{(\mathrm{l})}= & \int_{V_{i}}\left(\boldsymbol{B}_{i}(\boldsymbol{x}) \boldsymbol{C}_{i}\right)^{\mathrm{T}} \overline{\boldsymbol{D}} \boldsymbol{B}_{i}(\boldsymbol{x}) \boldsymbol{C}_{i} d V_{i}(\boldsymbol{x}) \\
\boldsymbol{K}_{i}^{(\mathrm{nl})}= & \frac{1}{2} \sum_{j=1}^{n} \int_{V_{i}} \int_{V}\left\{\boldsymbol{N}(\boldsymbol{\xi}) \boldsymbol{C}-\boldsymbol{N}_{i}(\boldsymbol{x}) \boldsymbol{C}_{i} \cdot\right\}^{\mathrm{T}} \boldsymbol{G}(\boldsymbol{x}, \boldsymbol{\xi}) \\
& \times\left\{\boldsymbol{N}(\boldsymbol{\xi}) \boldsymbol{C}-\boldsymbol{N}_{i}(\boldsymbol{x}) \boldsymbol{C}_{i}\right\} \mathrm{d} V(\boldsymbol{\xi}) \mathrm{d} V_{i}(\boldsymbol{x})
\end{aligned}
$$

So that the total potential energy associated with the finite volume element $V_{i}$ reads:

$$
\Pi_{i}(\boldsymbol{d})=\frac{1}{2}\left(\boldsymbol{d}^{\mathrm{T}}\left(\boldsymbol{K}_{i}^{(\mathrm{l})}+\boldsymbol{K}_{i}^{(\mathrm{nl})}\right) \boldsymbol{d}\right)-\boldsymbol{f}_{i}^{\mathrm{T}} \boldsymbol{d}
$$


where the external load vector reads:

$$
\boldsymbol{f}_{i}=\int_{V_{i}} \boldsymbol{N}_{i}(\boldsymbol{x}) \boldsymbol{C}_{i} \overline{\boldsymbol{b}}(\boldsymbol{x}) \mathrm{d} V(\boldsymbol{x})+\int_{S_{i}} \boldsymbol{N}_{i}(\boldsymbol{x}) \boldsymbol{C}_{i} \overline{\boldsymbol{t}}_{\boldsymbol{n}}(\boldsymbol{x}) \mathrm{d} S_{i}(\boldsymbol{x})
$$

By introducing Equation (24) into Equation (16) the total potential energy is provided as:

$$
\Pi=\Pi(\boldsymbol{d})=\frac{1}{2}\left(\boldsymbol{d}^{\mathrm{T}}\left(\boldsymbol{K}^{(\mathrm{l})}+\boldsymbol{K}^{(\mathrm{nl})}\right) \boldsymbol{d}\right)-\boldsymbol{f}^{\mathrm{T}} \boldsymbol{d}
$$

and

$$
\begin{aligned}
\boldsymbol{K}^{(\mathrm{l})} & =\sum_{i=1}^{n} \boldsymbol{K}_{i}^{(\mathrm{l})} \\
\boldsymbol{K}^{(\mathrm{nl})} & =\sum_{i=1}^{n} \sum_{j=1}^{n} \boldsymbol{K}_{i j}^{(\mathrm{nl})}
\end{aligned}
$$

where

$$
\begin{aligned}
\boldsymbol{K}_{i l}^{(\mathrm{nl})=} & \frac{1}{2} \int_{V_{i}} \int_{V_{j}}\left\{\boldsymbol{N}(\boldsymbol{\xi}) \boldsymbol{C}-\boldsymbol{N}_{i}(\boldsymbol{x}) \boldsymbol{C}_{i}\right\}^{\mathrm{T}} \boldsymbol{G}(\boldsymbol{x}, \boldsymbol{\xi}) \\
& \times\left\{\boldsymbol{N}(\boldsymbol{\xi}) \boldsymbol{C}-\boldsymbol{N}_{i}(\boldsymbol{x}) \boldsymbol{C}_{i}\right\} \mathrm{d} V(\boldsymbol{\xi}) \mathrm{d} V_{i}(\boldsymbol{x})
\end{aligned}
$$

It is worthy noticing that the non-local stiffness matrix $\boldsymbol{K}^{(\mathrm{nl})}$ is a fully populated, symmetric and positive-definite matrix. The character of positive definition of the stiffness matrix $\boldsymbol{K}^{(\mathrm{nl})}$ is fully accomplished as long as the strong formulation of the Drucker stability principle is fulfilled in the whole domain (see [41] for more details). The elastic equilibrium problem involving the solution of the nodal displacement vector $\boldsymbol{d}$ of the mesh grid is obtained by the condition:

$$
\delta \Pi=\frac{\partial \Pi}{\partial \boldsymbol{d}} \delta \boldsymbol{d}=0 \quad \forall \delta \boldsymbol{d} \Rightarrow \boldsymbol{K} \boldsymbol{d}-\boldsymbol{f}=0
$$

where we introduced the total stiffness matrix of the considered elastic solid in the form:

$$
\boldsymbol{K}=\boldsymbol{K}^{(\mathrm{l})}+\boldsymbol{K}^{(\mathrm{nl})}
$$

At this stage some comments involving the non-local stiffness matrix represented in Equation (27) are necessary. In fact the band of the stiffness matrix $\boldsymbol{K}$ is larger than the band of the stiffness matrix containing only the local terms $\boldsymbol{K}^{(\mathrm{l})}$. This is due to the presence of non-vanishing terms $K_{i j}^{(\mathrm{nl})}=K_{j i}^{(\mathrm{nl})}$ that represents the stiffness of the long-range bonds between elements $V_{i}$ and $V_{j}$ so that the computational efforts to solve by numerical inversion the algebraic system in Equation (29) may become very large. Those contributions are, however, rapidly vanishing with the interdistance of the interacting elements and thus it is convenient, for numerical purposes, the introduction of a central parameter $L_{R}$ that represent the radius of the horizon of significative long-range effects (Figure 2). In this context an FE library may be built accounting for self-stiffness contributions $K_{i i}^{(\mathrm{nl})}$ and only a subset of cross-terms $K_{i j}^{(\mathrm{nl})}$ conveniently chosen and depending on the relative distance between the couple of element considered. In this context an optimization of the computer code may be obtained allowing for a significative time-saving in the assembly of non-local stiffness matrices.

\section{NUMERICAL APPLICATIONS}

The proposed FEM to solve the elastic problem posed within the mechanically based model of nonlocal elasticity has been applied in this section two cases: (i) An 1D elastic bar in simple traction; (ii) A plane elasticity problem involving a squared domain under external boundary tractions. Both cases have been contrasted with the solution obtained with approximate analytical methods used 


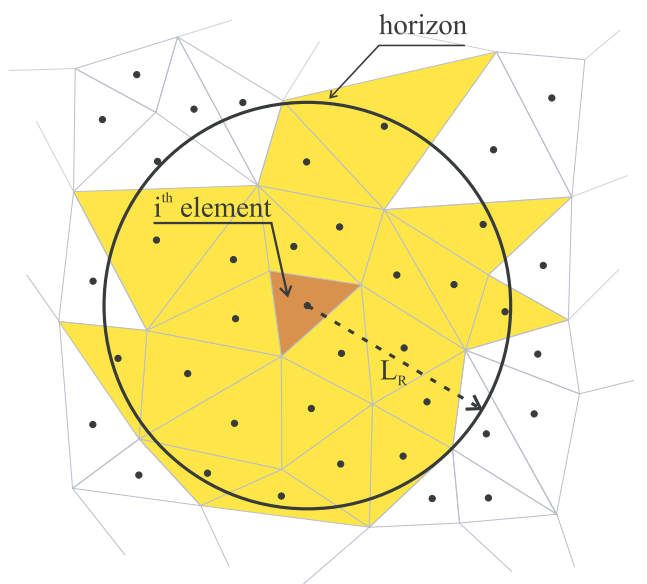

Figure 2. Triangular elements with centroids. Matrices $\boldsymbol{K}_{i l}^{(\mathrm{nl})}$ relative to yellow elements are computed for the evaluation of the element matrix $\boldsymbol{K}_{i}^{(\mathrm{nl})}$.

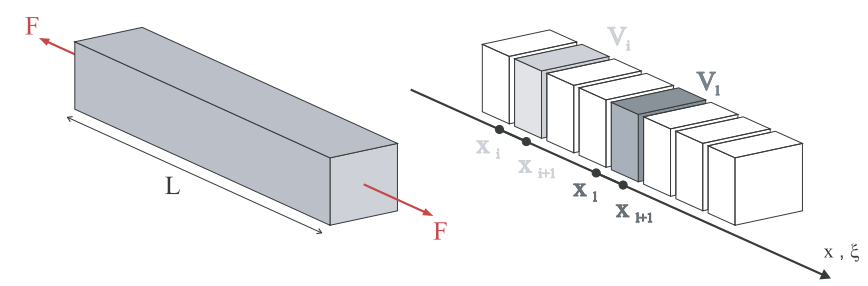

Figure 3. Mesh of a 1D bar.

by the authors in previous papers. The numerical codes used have been structured as shown in Algorithm (1): from line $\mathbf{1}$ to $\mathbf{8}$, the code evaluates, for any element $i(i=1,2, \ldots, n)$, the elements within the horizon radius $L_{R}$ by means of the distance between their centroids $x_{i}^{G}$ and $x_{j}^{G}$, and collects them in the array NeighElm(i). In line 10, elements of the global stiffness matrix are initialized to zero and, from line $\mathbf{1 1}$ to $\mathbf{1 9}$, two nested loops assemble the global stiffness matrix $\boldsymbol{K}$ and the load vector $\boldsymbol{f}$; then, a sub-routine called FindUnknown () supplies an array collecting indices of unknown displacements in order to condition the stiffness matrix for the solution.

\subsection{D elastic bar in tension}

Let us consider an elastic bar of length $L=20 \mathrm{~cm}$, with uniform cross-section $A=1 \mathrm{~cm}^{2}$ and Young's modulus $E=2.110^{6} \mathrm{daNcm}^{-2}$, loaded with a self-equilibrated couple of external loads $F=100 \mathrm{daN}$. The bar, depicted in Figure 3, is subdivided along the direction $x$ in $n$ elements of constant length $\Delta x$ with nodes coordinates $x_{i}=i \Delta x$ and $x_{i+1}=(i+1) \Delta x$. The FEM solution has been obtained assuming a linear displacement field so that the vector of the shape function is provided in the form:

$$
\boldsymbol{N}^{(i)}(\boldsymbol{x})=\left(\begin{array}{ll}
\frac{x_{i+1}-x}{x_{i+1}-x_{i}} & \frac{x-x_{i}}{x_{i+1}-x_{i}}
\end{array}\right)
$$

and, considering Equation (20a)

$$
\boldsymbol{B}^{(i)}(\boldsymbol{x})=\left(\begin{array}{ll}
-\frac{1}{x_{i+1}-x_{i}} & \frac{1}{x_{i+1}-x_{i}}
\end{array}\right)
$$




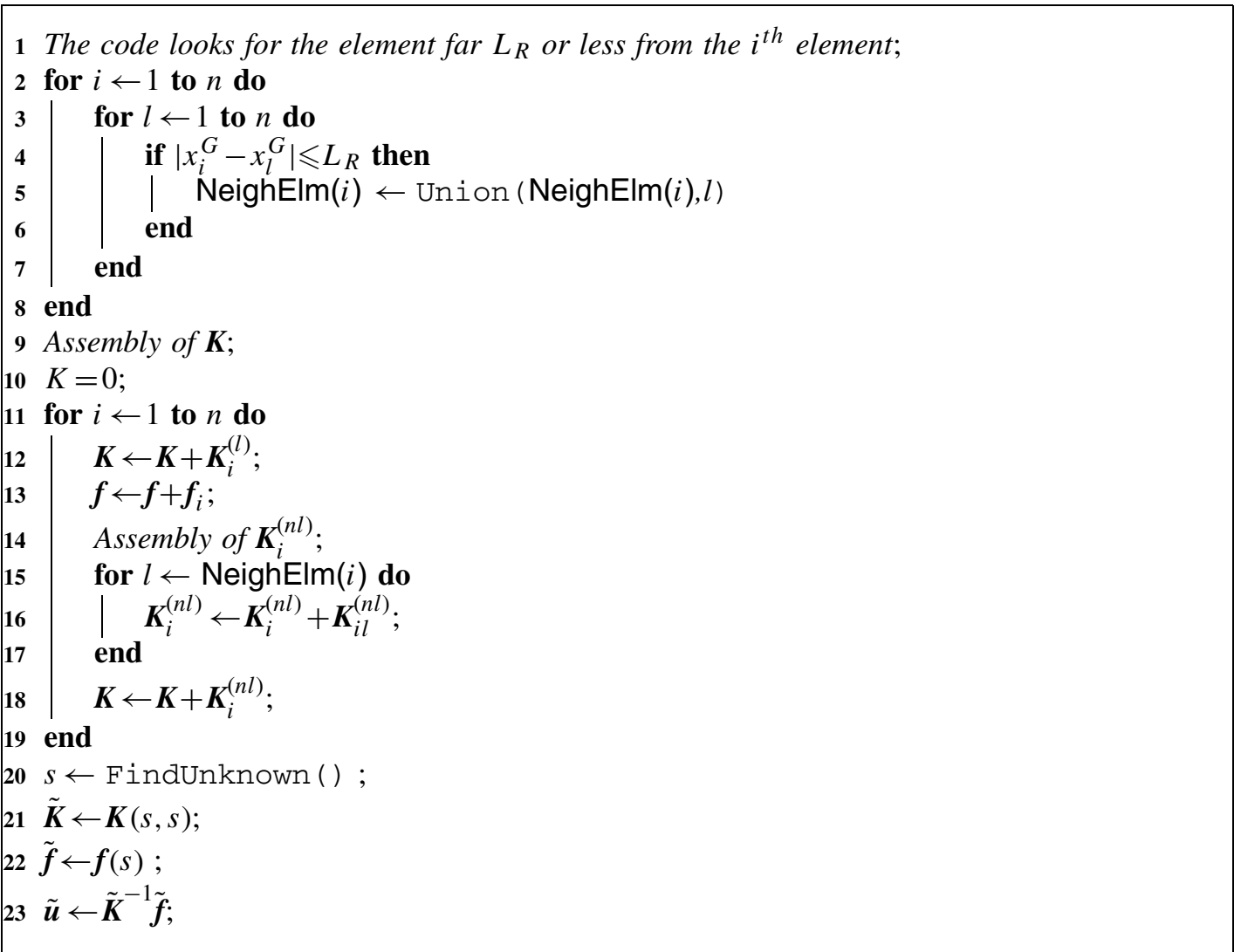

Algorithm 1: Non-local FEM procedure.

The decay function, according to [41], has been chosen in the form:

$$
g(x, \xi)=\frac{\beta E}{2 \ell A^{2}} \mathrm{e}^{-|x-\xi| \ell^{-1}}
$$

In order to compare the results with the ones supplied by the finite difference code developed previously [43], for this 1D problem, all the non-local element matrix $\boldsymbol{K}_{i j}^{(\mathrm{nl})}$ will be considered corresponding to $L_{R} \rightarrow \infty$. The numerical results obtained with the FEM code have been represented in Figures 4(a) and (b). In Figure 4(a) the displacement field along the bar has been reported for different values of the internal length $\ell$. It may be observed that, as far as the internal length increases, a stiffer model is involved and stronger non-local effects may be detected. This is mechanically due to the consideration that more and more springs are involved in the model as far as the internal length $\ell$ increases.

The estimation of the relative error involved in the FEM discretizaton has been evaluated resorting to measure of the elastic potential energy of the bar. To this aim in Figure 4(b), we represented a non-dimensional ratio $|\Phi-\bar{\Phi}| / \bar{\Phi}$ vs number of elements in the mesh where $\Phi$ is the elastic potential energy of the discretized model and $\bar{\Phi}$ is the total elastic energy calculated with an FEM solution of 2000 elements and assumed as benchmark solution . The observation of Figure 4 shows that in all the cases analyzed the FEM achieves faster convergence with respect to FDM. The additional computational effort to evaluate displacements, stress and strains within FEM for non-local mechanics is related only to the construction of the non-local stiffness matrix $\boldsymbol{K}^{(\mathrm{nl})}$ since for each element we need to evaluate the local stiffness matrix $\boldsymbol{K}_{i}^{(\mathrm{l})}$ and $n$ stiffness matrices $\boldsymbol{K}_{i j}^{(\mathrm{nl})}$ (Figure 5). 

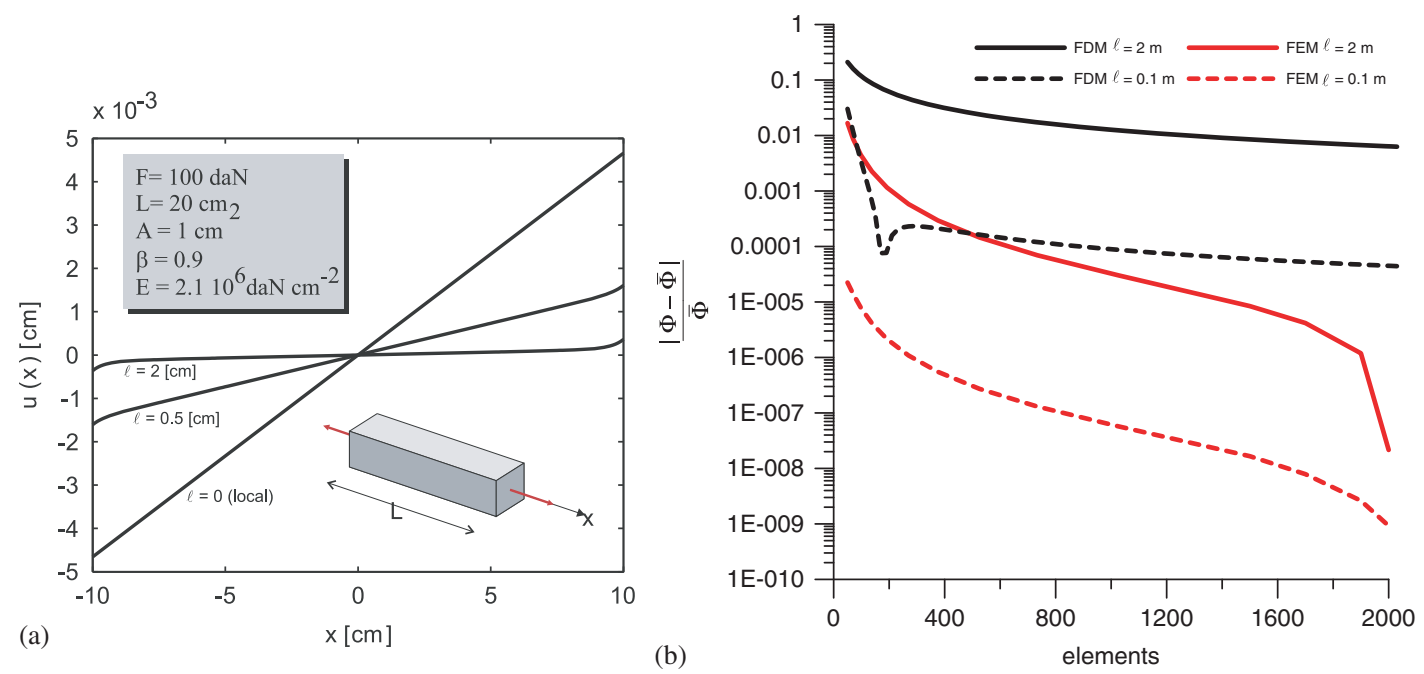

Figure 4. Axial displacement (a) Elastic potential energy convergence for different $\ell$ ( $\beta=0.9$ ) (b).

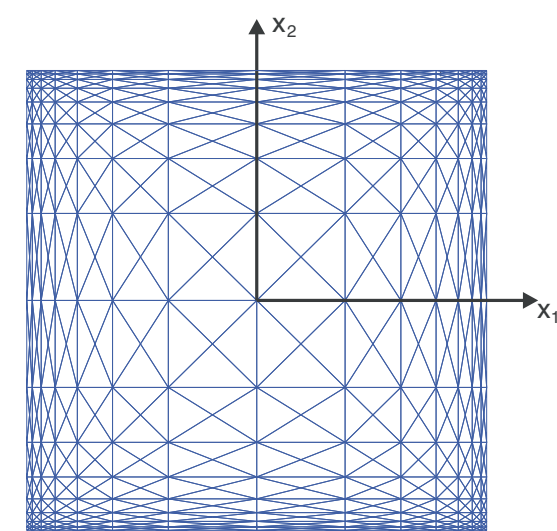

(a)

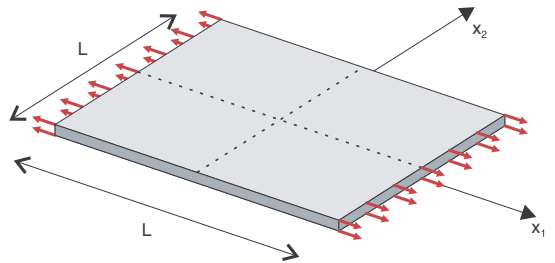

(b)

Figure 5. Eight hundred and ninety-six elements FE mesh (479 nodes—940 DOF) (a); square plate in plain stress state (b).

\subsection{A $2 D$ plane elasticity problem}

Let us consider a squared elastic layer of edge $L$, in plane stress state, loaded by a self-equilibrated system of constant traction $\sigma_{11}( \pm 0.5 \cdot L, y)=50 \times 10^{3} \mathrm{daNm}^{-2}$ on two opposite edge and with mechanical parameters selected to $E=2.1 \times 10^{10} \mathrm{daNm}^{-2}, v=0.3$. The decaying function is assumed as

$$
g(\boldsymbol{x}, \boldsymbol{\xi})=C \mathrm{e}^{-|\boldsymbol{x}-\boldsymbol{\xi}| \ell^{-1}}
$$

where $C=10^{14} \mathrm{daNm}^{-2}$ and $\ell=0.1 \mathrm{~m}$ as reported in [41]. Numerical results have been compared with those supplied by the FDM and Galerkin approximations used in previous papers [43] and henceforth no horizon have been included $\left(L_{R} \rightarrow \infty\right)$ including all the elements in the evaluation of the non-local stiffness matrix $\boldsymbol{K}^{(\mathrm{nl})}$. The domain has been meshed with triangular linear elements with 3 Gauss integration points and the result obtained have been contrasted with a Galerkin solution and an FDM one [41] and the mesh grid has been refined near to the boundaries to highlight the higher gradients of the displacement function. The program developed in MATLAB ${ }^{\circledR}$ to implement the FEM ran for $2577 \mathrm{~s}$ to compute the solution, whereas the FDM program used in [41] ran for $4372 \mathrm{~s}$ on the same machine and operating system. Figure 6 shows that FEM solution 

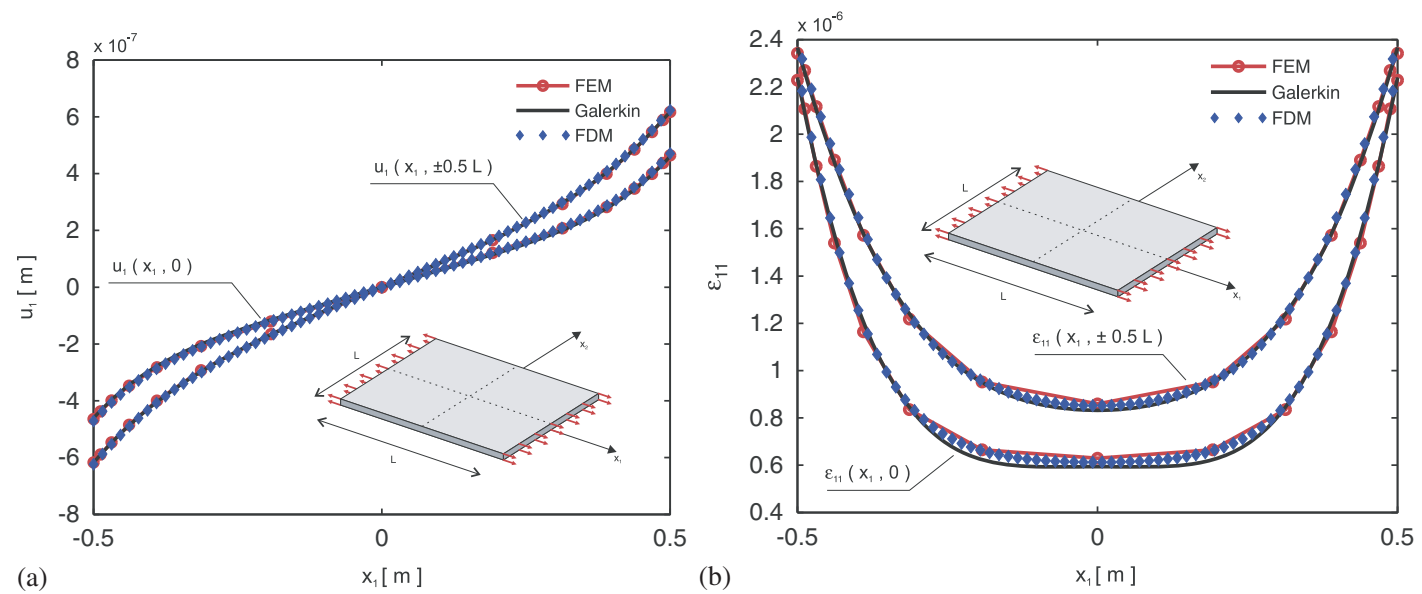

Figure 6. (a) Displacement component $u_{1}$ and (b) strain component $\varepsilon_{11}$ along section planes parallel to the $x_{1}$ axis.
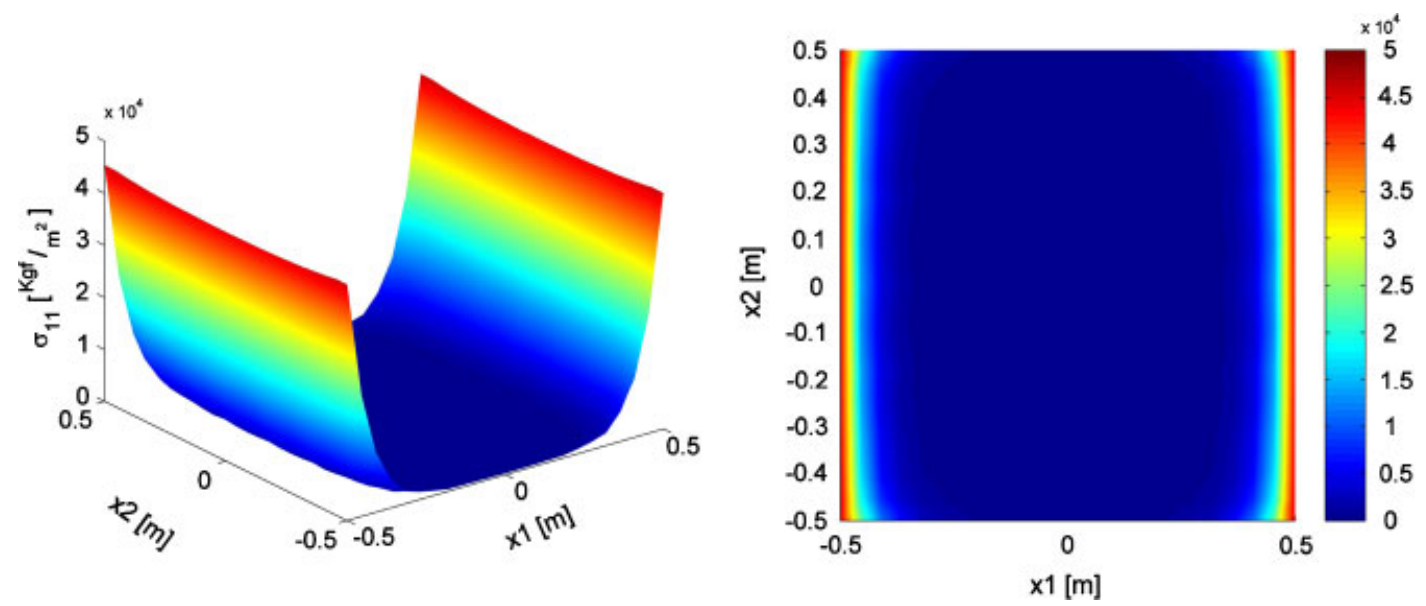

Figure 7. $\sigma_{11}\left(x_{1}, x_{2}\right)$ for $\ell=0.5$.

totally fits the others (FDM and Galerkin) which, together with the shorter computing time, shows the efficiency of the algorithm proposed. The stress fields $\sigma_{11}$ and $\sigma_{12}$ have been reported in Figures 7 and 8 of the $\sigma_{i j}\left(x_{1}, x_{2}\right)$ for a prescribed value of the internal length $\ell=0.5 \mathrm{~mm}$.

The introduction of the horizon parameter $L_{R}$ enhances the effciency of the FEM code as expected; In fact assuming that, for example, the horizon radius proportional to the internal length $\ell L_{R}=\ln (400) \ell$ or $L_{R}=\ln (100) \ell$ the reduction of the computational time is, respectively, of $76 \%$ and of $84 \%$ with respect to the absence of the horizon. In fact in this latter case all the matrices $\boldsymbol{K}_{i j}^{(\mathrm{nl})}$ must be evaluated. A parametric study of the influence of the horizon radius $L_{R}$ may be performed introducing the ratio:

$$
r_{g}=\frac{g(0)}{g\left(L_{R}\right)}=\mathrm{e}^{L_{R} / \ell}
$$

so that we may evaluate the changes of the total elastic energy, measured by the ratio $|\Phi-\bar{\Phi}| / \bar{\Phi}$ for different values of $r_{g}$ as reported in Figure 9. When $r_{g} \approx 100$ the order of magnitude of the relative error is very small $\left(10^{-3}\right)$ although the solution shows small but still visible differences on the core of the squared domain. Several other numerical applications with higher-order interpolation of the displacement field and Gauss integration points have been investigated. More precisely, elements 


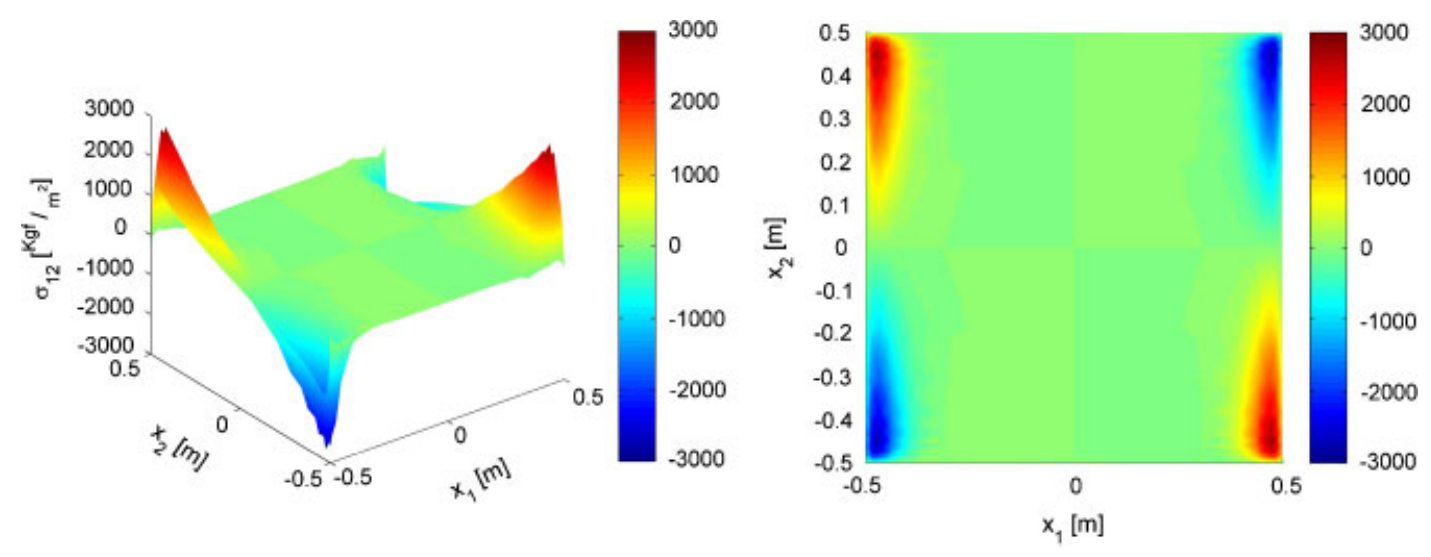

Figure 8. $\sigma_{12}\left(x_{1}, x_{2}\right)$ for $\ell=0.5$.

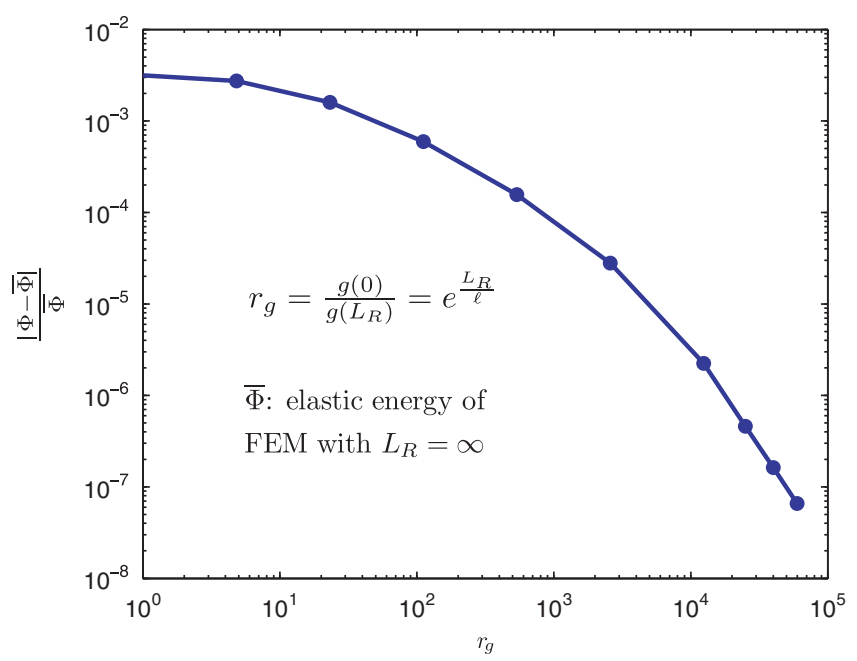

Figure 9. Relative error of the internal elastic energy versus $r_{g}$ parameter.

with 4 and 7 Gauss points have been used. The time required to solve the 2D test problem is 2577 s with 3 Gauss points, 6363 s with 4 Gauss points and 10941 s with 7 Gauss points. Anyway, Figure 10(a) shows that the gain in precision is quite small when higher polynomials are employed for the integration of the stiffness matrices; hence, in our opinion, 3 Gauss points elements are a reasonable choice in order to achieve good precision and faster computations. Furthermore, the mechanically based model has been tested also with a 8-node QUAD element FEM whose results are shown in Figure 10(b). The comparison here proposed is between a 896 element solution with 3-node triangular elements with 3 Gauss points and a 400 element regular mesh solution with 8-node serendipity elements with 9 Gauss points. However, the increasing precision of the latter solution with a consistent increment of the computational time corresponds to very small differences in the accuracy of the result with respect to the rough triangular mesh originally used.

In authors' opinion, this is due to the intrinsically mechanical representation of non-local effects as long-range forces that in this theory they depends only on the relative displacement field; so that, as far as displacement field is accurate enough, no significant error is included in the nonlocal terms. This is an important issue of the used non-local theory since it allows to formulate a FEM code for non-local problems in standard fashion with high accuracy yet with single linear elements. 

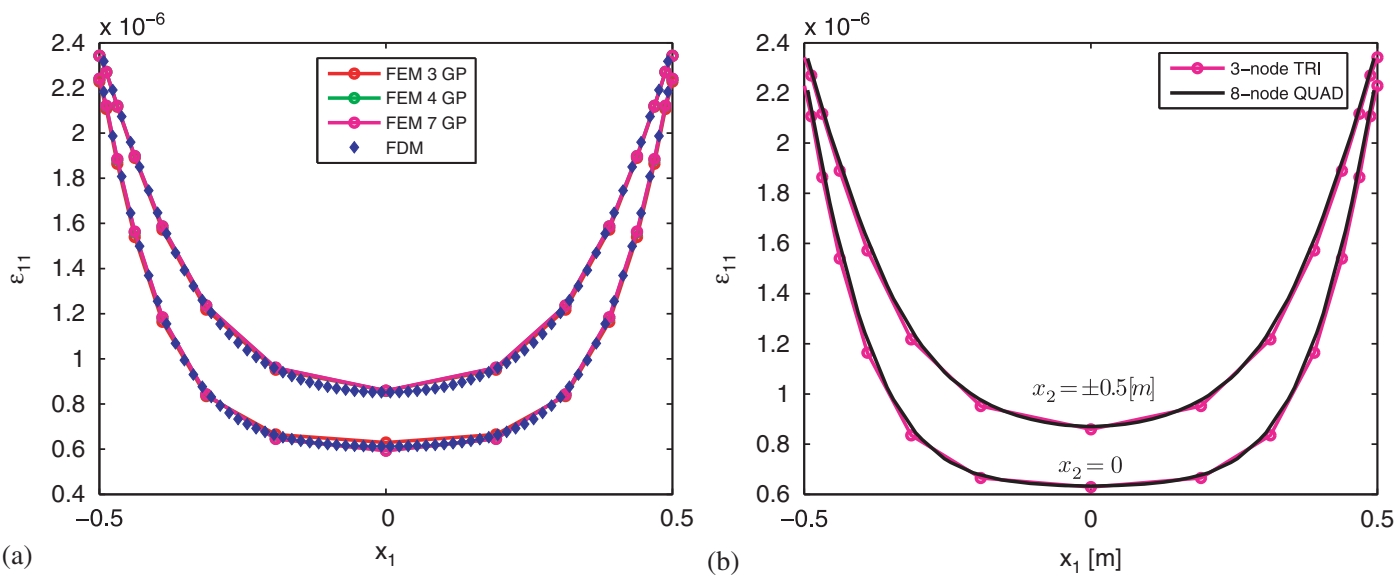

Figure 10. Comparison of the strain component $\varepsilon_{11}$ along section planes parallel to the $x_{1}$ axis among different numbers of Gauss points (a) and between triangular and 8-node quadrangular elements (b).

As the validation of the proposed FEM code to analyze non-local systems in the presence of long-range interactions has been assessed by the previous considerations, a numerical comparison with the Eringen integral model of non-local elasticity for the plane elasticity problem illustrated in Figure 5(b) has been reported. The numerical solution of Eringen's model of non-local elasticity has been obtained by means of a finite element code with 8-node serendipity rectangular elements and 9 Gauss points (see e.g. [16] for details). In fact, the triangular finite element code that has been used for the mechanically based model yield a too rough solution as applied to Eringen's model. The stress field $\sigma_{11}$ and the $\varepsilon_{11}$ strain field have been evaluated for both models and for different values of the internal length $\ell$. In the mechanically based model of non-local elasticity, the stress field coincides with the classical Cauchy local stress whereas in Eringen's approach the stress field is a non-local field, dubbed as $\hat{\boldsymbol{\sigma}}(\boldsymbol{x})$, and defined as follows [16]:

$$
\hat{\boldsymbol{\sigma}}(\boldsymbol{x})=\zeta_{1} \boldsymbol{D}: \boldsymbol{\varepsilon}(\boldsymbol{x})+\zeta_{2} \int_{V} g(|\boldsymbol{x}-\boldsymbol{\xi}|) \boldsymbol{D}: \boldsymbol{\varepsilon}(\boldsymbol{\xi}) \mathrm{d} V(\boldsymbol{\xi}),
$$

where $\boldsymbol{D}$ is the elastic fourth-rank tensor, $\boldsymbol{\varepsilon}$ is the strain tensor, $\zeta_{1}$ and $\zeta_{2}$ are the volume fractions of the local and non-local material, respectively, such that $\zeta_{1}+\zeta_{2}=1$. Function $g(|\boldsymbol{x}-\boldsymbol{\xi}|)$ is the attenuation kernel of Eringen's model that has been selected, for the sake of numerical comparison, in the same functional class of 34 that reads [16]:

$$
g(|x-\xi|)=\frac{1}{2 \pi \ell^{2} s} \mathrm{e}^{-|x-\xi| \ell^{-1}}
$$

where $s$ is the plate thickness. In the reported numerical comparison we have selected $\zeta_{1}=\zeta_{2}=0.5$ according to [16]. In order to evaluate the stress field for each Gauss point, Equation (36) can be written as follows:

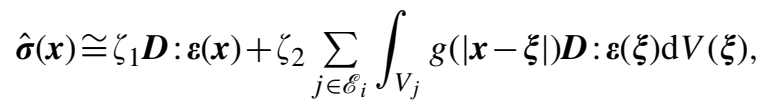

where $\mathscr{E}_{i}$ is the set of elements whose centroid is far or less than $L_{R}=6 \ell$ from centroid of the $i$ th element. In fact, as for the non-local matrix assembly, it is fair to assume that also the evaluation of the stress field of Eringen's model can be simplified neglecting elements further than a prescribed distance, namely $L_{R}$, which depends on the internal length $\ell$. Hence, approximating the integral of the $j$ th element volume $V_{j}$ in Equation (38) we obtain

$$
\hat{\boldsymbol{\sigma}}\left(\boldsymbol{x}_{i}^{(k)}\right)=\zeta_{1} \boldsymbol{D}: \boldsymbol{\varepsilon}\left(\boldsymbol{x}_{i}^{(k)}\right)+\zeta_{2} \sum_{j \in \mathscr{E}_{i}} \sum_{l=1}^{9} g\left(\left|\boldsymbol{x}_{i}^{(k)}-\boldsymbol{x}_{j}^{(\mathrm{l})}\right|\right) \boldsymbol{D}: \boldsymbol{\varepsilon}\left(\boldsymbol{x}_{j}^{(\mathrm{l})}\right) V_{j} w(l),
$$



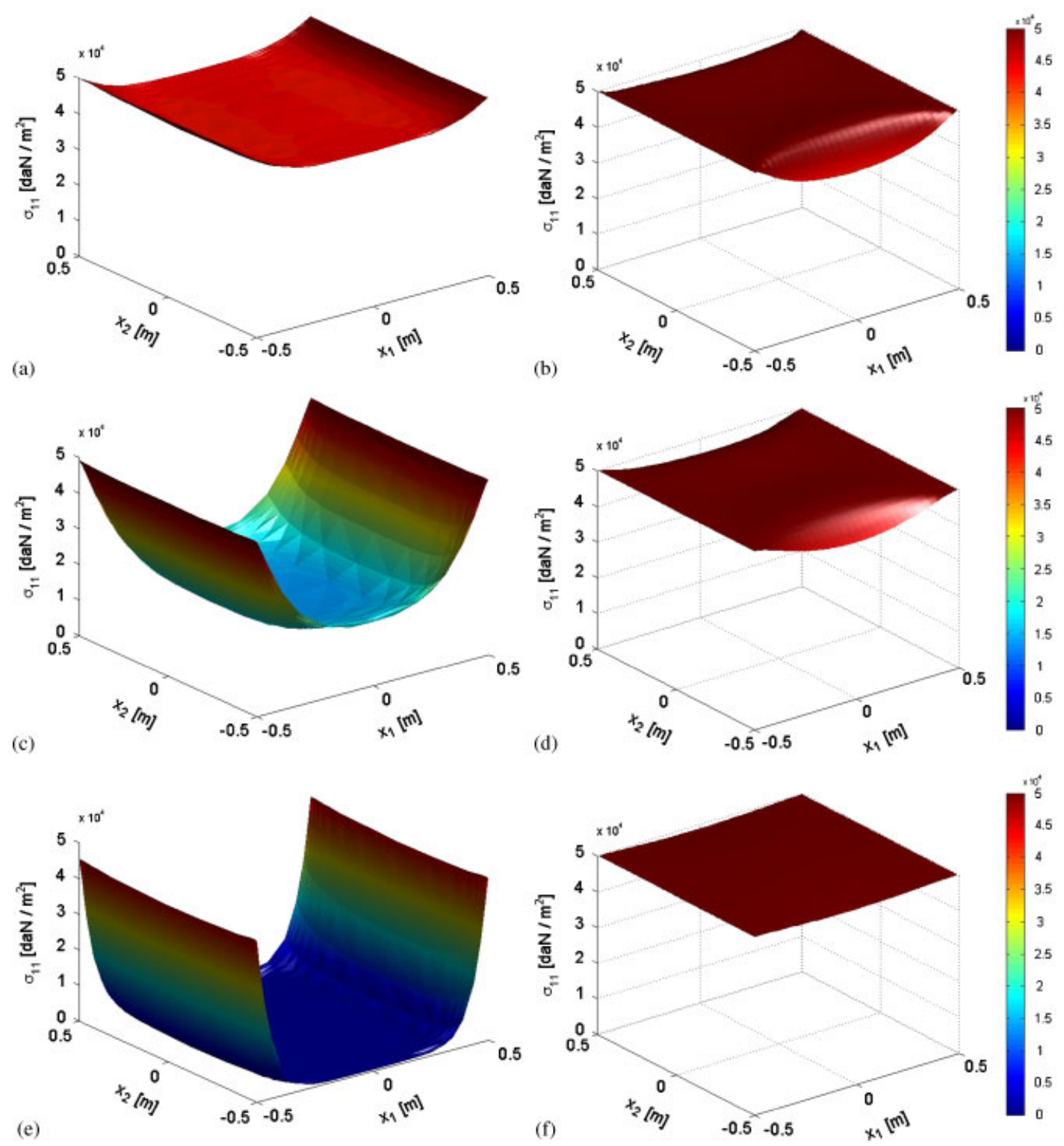

Figure 11. $\sigma_{11}\left(x_{1}, x_{2}\right)$ plots of the study case of Figure 5(b) for different values of $\ell$ : (a) mechanically-based $\ell=0.05[\mathrm{~m}]$; (b) eringen $\ell=0.05[\mathrm{~m}]$; (c) mechanically-based $\ell=0.1[\mathrm{~m}]$; (d) eringen $\ell=0.1[\mathrm{~m}]$;

(e) mechanically-based $\ell=0.5[\mathrm{~m}]$; and (f) eringen $\ell=0.5[\mathrm{~m}]$.

with $\boldsymbol{x}_{i}^{(k)}$ being the coordinate of the $k$ th Gauss point of the $i$ th element and $w(l)$ the weight coefficient related to the $l$ th Gauss point of the generic element. The stress field pattern is shown in Figure 11 both for Eringen and the mechanically based model of non-local elasticity. The Eringen model has been analyzed by [21] FEM method as discussed in Equations (36)-(39).

It may be observed that contrasting the stress field for different values of the internal length scale $\ell$, for the mechanically based model, the non-local behavior is vanishing as the internal length decreases, recovering the constant value on the entire domain. Eringen's model instead tends to recover the local solution only in the inner part of the domain and on the loaded edge while it exhibits lower values on the two opposite free edges which become more marked as $\ell$ decreases. The strain field $\varepsilon_{11}$ corresponding to the mechanically based and the Eringen model of non-local elasticity have been reported in Figure 12 for different values of the internal length. It may be observed that, in the mechanically based model the local solution is recovered as $\ell \rightarrow 0$ 

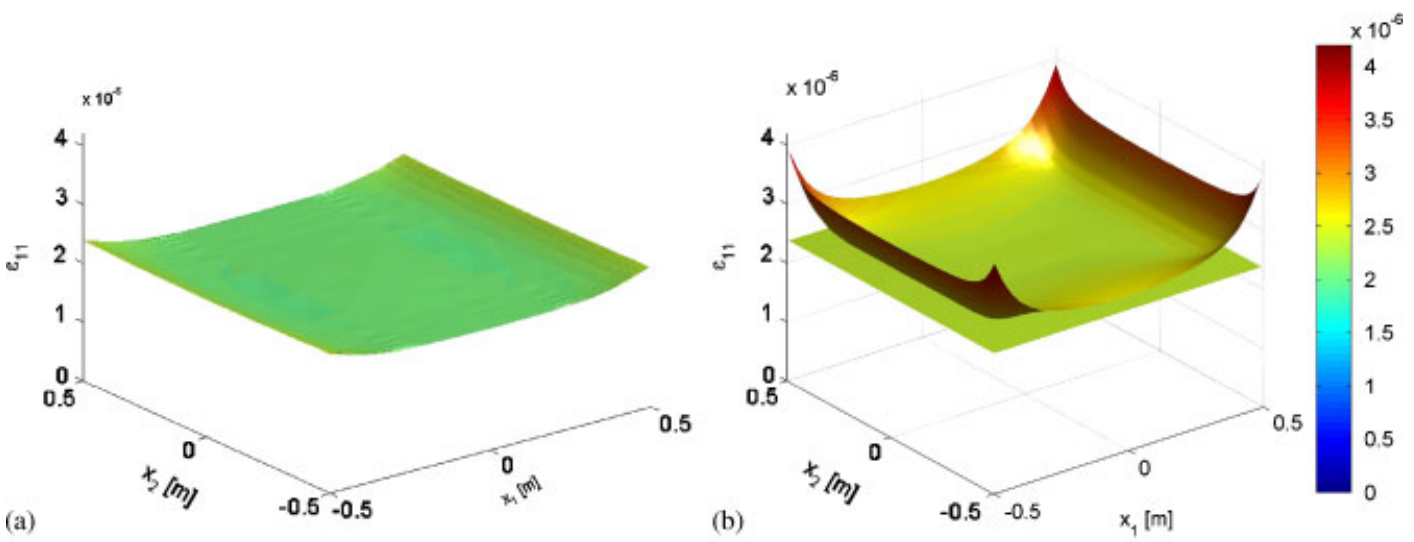

(a)

(b)
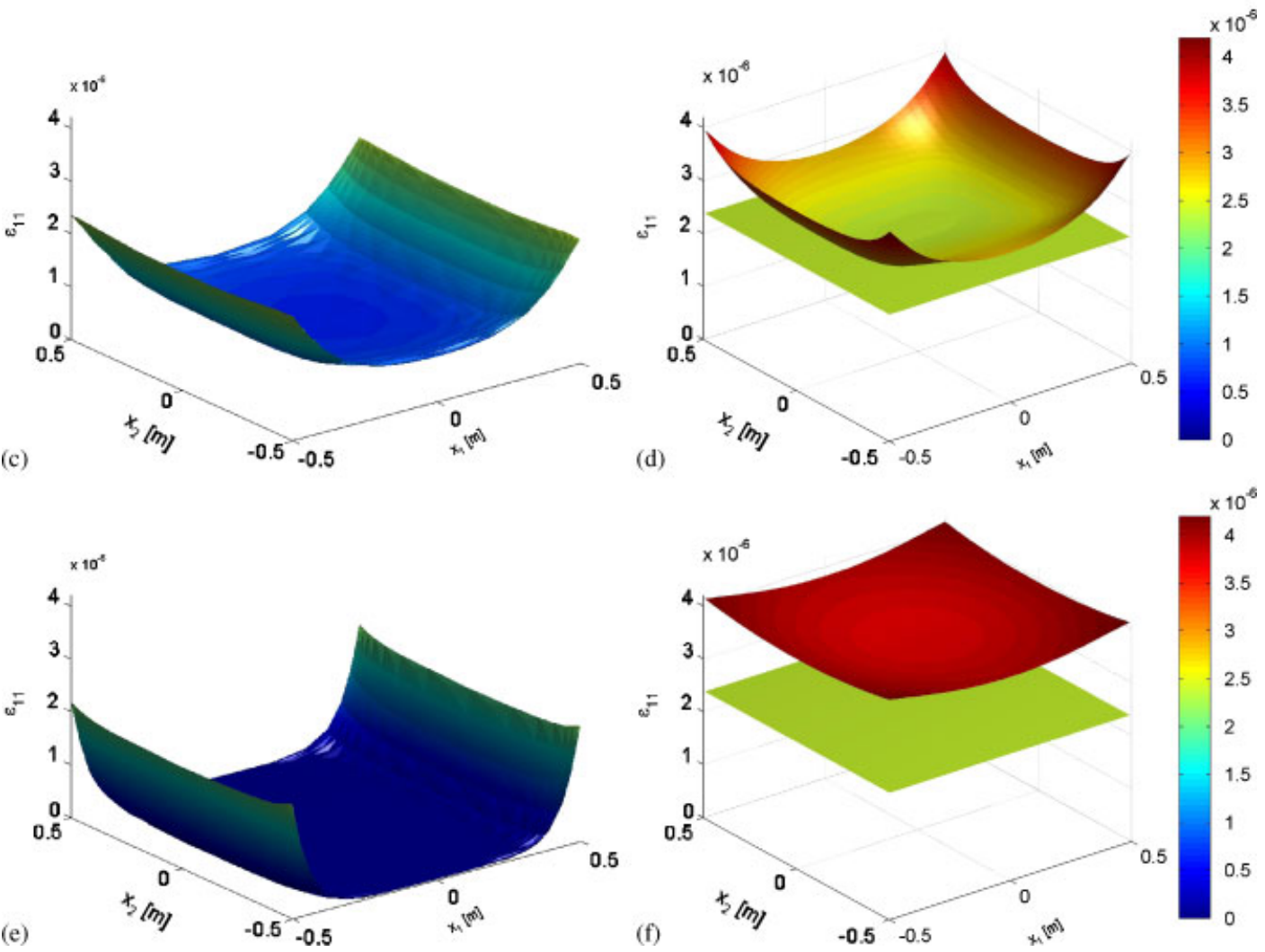

Figure 12. $\varepsilon_{11}\left(x_{1}, x_{2}\right)$ plots of the study case of Figure 5 for different values of $\ell$ : (a) mechanically-based $\ell=0.05[\mathrm{~m}]$; (b) eringen $\ell=0.05[\mathrm{~m}]$ and local solution; (c) mechanically-based $\ell=0.1[\mathrm{~m}]$; (d) eringen $\ell=0.1[\mathrm{~m}]$ and local solution; (e) mechanically-based $\ell=0.5[\mathrm{~m}]$; and

(f) eringen $\ell=0.5[\mathrm{~m}]$ and local solution.

since the strain is just a linear combination of local stress fields. In Eringen's model, instead, the local solution of classical elasticity is only partially recovered in the core domain showing higher values on the edges with peaks on the corners.

Even if there are no experimental results which can establish the absolute accuracy of the mechanically based model among others, in the authors' opinion the model proposed here actually simplifies the position of boundary conditions with respect to other non-local models and it shows a complete consistency over the class of decay function that are symmetric and strictly positive. The Eringen model, on the other hand, exhibits a different behavior of the solution close to the borders of the domain. This, in the authors' opinion, means that the Eringen model may be used to 
describe phenomenological behaviors which are different form those predicted by the mechanically based model, although both of them are non-local in nature.

\section{CONCLUSIONS}

The paper has been devoted to the formulation of an FEM for non-local problems that is consistent with the mechanically based non-local elasticity theory, recently proposed. The method has been developed formulating the elastic equilibrium problem in weak form by means of the total potential energy functional. As in classical FEM a discretization of the domain and the approximation of the displacement function in each subdomain yields the algebraic equations of the FEM in the unknowns reported by nodal displacements. The matrix coefficients of the algebraic system report local and non-local contributions and both are symmetric and positive definite (upon the application of boundary conditions).

The numerical studies handled in the paper have been reported to challenge the capabilities of the FEM with respect to other solution methods used in previous studies. The analysis has been carried out in 1D and 2D cases and several types of finite elements with different orders of the interpolation of the unknown function have been used. All the analysis have shown that the use of higher-order in interpolation of the displacement fields does not involve an appreciable increase of the accuracy of the solution with respect to the use of linear triangular finite elements. Therefore, only the results obtained with triangular finite elements have been reported in the paper contrasted with the solution obtained via other approximate methods. It has been shown that the solution obtained with the proposed FEM is more convenient with respect to FDM or other approximate methods both in the $1 \mathrm{D}$ and in the $2 \mathrm{D}$ cases studied.

In the 1D case the terms involved in the evaluation of FE non-local matrices may be obtained in closed-form whereas in the higher-dimensional domain an approximate evaluation of integrals via Gauss quadrature methods must be used. The numerical studies conducted shown that the order of the quadrature does not affect the quality of the solution and henceforth a coarse 3 Gauss points quadrature may be considered without a significative loss of accuracy in the numerical results. This aspect is closely related to the mechanically based model of non-local elasticity that introduces the long-range forces in terms of the relative displacements between interacting volumes. Therefore, as far as a sufficiently accurate representation of the displacement field in the solid is achieved, as with linear triangular finite elements, no significative enhancement of the accuracy of the FEM solution is obtained for higher-order quadrature. Similar considerations hold true also in terms of the order of the approximation of the displacement field within the finite element used.

We therefore conclude that the proposed FEM is a convenient choice to handle non-local problems in the context of the mechanically based model of non-local elasticity.

\section{ACKNOWLEDGEMENTS}

This study has been conducted under the auspices of the Italian Ministry of Scientific and Technological Research through program PRIN2008 (National Coordinator Prof. Carpinteri). This financial support is gratefully acknowledged.

\section{REFERENCES}

1. Born M, Huang K. Dynamical Theory of Crystal Lattices. Oxford University Press: London, 1954.

2. Cosserat E, Cosserat F. Thorie des corps dformables Librairie Scientifique A. Hermann et Fils: Paris, 1909.

3. Ericksen JL, Truesdell C. Exact theory of stress and stress and strain in rods and shell. Archive for Rational Mechanics and Analysis 1957; 1:295-323.

4. Toupin RA. Elastic materials with couple stresses. Archive for Rational Mechanics and Analysis 1962; 11:385-414.

5. Mindlin RD. Micro-structure in linear elasticity. Archive for Rational Mechanics and Analysis 1964; 16:51-78.

6. Mindlin RD, Eshel NN. On first strain-gradient theories in linear elasticity. International Journal of Solids and Structures $1968 ; 4: 109-124$. 
7. Kröner E. Elasticity theory of materials with long range cohesive forces. International Journal of Solids and Structures 1967; 3:731-742.

8. Eringen AC. Linear theory of non-local elasticity and dispersions of plane waves. International Journal of Engineering Science 1972; 10:425-435.

9. Kunin IA. Elastic Media with Microstructure I: One-dimensional Models. Springer Series in Solid-State Sciences, vol. 44. Springer: Berlin, Heidelberg, New York, 1982.

10. Kunin IA. Elastic Media with Microstructure II: Three-dimensional Models. Springer Series in Solid-State Sciences, vol. 44. Springer: Berlin, Heidelberg, New York, 1983.

11. Hughes TJR. Multiscale phenomena: Green's functions, the Dirichlet-to-Neumann formulation, subgrid scale models, bubbles and the origins of stabilized methods. Computer Methods in Applied Mechanics and Engineering 1995; 127:387-401.

12. Garikipati K, Hughes TJR. A variational multiscale approach to strain localization-formulation for multidimensional problems. Computer Methods in Applied Mechanics and Engineering 2001; 188:39-60.

13. Trovalusci P, Masiani R. Non-linear micropolar and classical continua for anisotropic discontinuous materials. International Journal of Solids and Structures 2003; 40:1281-1297.

14. Picu C. A nonlocal formulation of rubber elasticity. International Journal for Multiscale Computational Engineering 2003; DOI: 10.1615/IntJMultCompEng.v1.i1.30.

15. Aliabadi MH. Multi-scale boundary element modelling of material degradation and fracture. Computer Methods in Applied Mechanics and Engineering 2007; 196:1310-1329.

16. Eringen AC, Edelen DGB. On non-local elasticity. International Journal of Engineering Science 1972; 10:233-248.

17. Eringen AC, Kim BS. Stress concentration at the tip of a crack. Mechanics Research Communications 1974; 1:233-237.

18. Mindlin RD, Eshel NN. On first strain-gradient theories in linear elasticity. International Journal of Solids and Structures 1968; 4:109-124.

19. Toupin RA. Theories of elasticity with couple-stress. Archive for Rational Mechanics and Analysis 1964; 17(2):85-112.

20. Pisano AA, Fuschi P. Closed form solution for a nonlocal elastic bar in tension. International Journal of Solids and Structures 2003; 40:13-23.

21. Pisano AA, Sofi A, Fuschi P. Nonlocal integral elasticity: 2D finite element based solutions. International Journal of Solids and Structures 2009; 46:3836-3849.

22. Benvenuti E, Tralli A. The fast Gauss transform for non-local integral FE models. Communications in Numerical Methods in Engineering 2005; 22:505-533.

23. Pijaudier-Cabot G, Bažant ZP. Nonlocal damage theory. Journal of Engineering Mechanics 1987; 113:1512-1533.

24. Polizzotto C, Borino G. A thermodynamics-based formulation of gradient-dependent plasticity. European Journal of Mechanics A/Solids 1998; 17:741-761.

25. Ganghoffer JF, De Borst R. A new framework in non-local mechanics. International Journal of Engineering Science 2000; 38:453-486.

26. De Sciarra FM. Variational formulations and a consistent finite-element procedure for a class of nonlocal elastic continua. International Journal of Solids and Structures 2008; 45:4184-4202.

27. Aifantis EC. Gradient effects at macro micro and nano scales. Journal of the Mechanical Behavior of Materials 1994; 5:355-375.

28. Altan BS, Aifantis EC. On some aspects in the special theory of gradient elasticity. Journal of the Mechanical Behavior of Materials 1997; 8:231-282.

29. Bažant ZP. Non local damage theory based on micromechanics of crack interactions. Journal of Engineering Mechanics (ASCE) 1994; 120(3):593-617.

30. Polizzotto C. Gradient elasticity and non standard boundary conditions. Journal of Solids and Structures 2003; 40:7399-7423.

31. Bažant ZP, Belytschko TB. Continuum theory for strain-softening. Journal of Engineering Mechanics 1984; 110(2):1666-1692.

32. Aifantis EC. Update on a class of gradient theories. Mechanics of Materials 2003; 35:259-280.

33. Silling SA. Reformulation of elasticity theory for discontinuities and long-range forces. Journal of the Mechanics and Physics of Solids 2000; 48:175-209.

34. Silling SA, Zimmermann M, Abeyaratne R. Deformation of a Peridynamic Bar. Journal of Elasticity 2003; 73:173-190.

35. Weckner O, Emmrich E. Numerical simulation of the dynamics of a nonlocal, inhomogeneous, infinite bar. Journal of Computational and Applied Mechanics 2005; 6:311-319.

36. Silling SA, Lehoucq RB. Convergence of peridynamics to classical elasticity theory. Journal of Elasticity 2008; 93:13-37.

37. Silling SA, Bobaru F. Peridynamic modeling of membranes and fibers. Computers and Structures 2005; 45: 395-409.

38. Jirásek M, Patzák B. Consistent tangent stiffness for nonlocal damage models. Computers and Structures 2002; 80:1279-1293.

39. Di Paola M, Zingales M. Long-range cohesive interactions of non-local continuum faced by fractional calculus. International Journal of Solids and Structures 2008; 45:5642-5659. 
40. Zingales M. Waves propagation in 1D elastic solids in presence of long-range central interactions. Journal of Sound and Vibrations 2010; DOI: 10.1016/j.jsv.2010.10.027.

41. Di Paola M, Failla G, Zingales M. Physically-based approach to the mechanics of strong non-local elasticity theory. Journal of Elasticity 2009; 97:103-130.

42. Di Paola M, Pirrotta A, Zingales M. Mechanically-based approach to non-local elasticity: variational principles. International Journal of Solids and Structures 2010; 47:539-548.

43. Di Paola M, Failla G, Zingales M. The mechanically-based approach to 3D non-local linear elasticity theory: long-range central interactions. International Journal of Solids and Structures 2010; 47:2347-2358.

44. Di Paola M, Marino F, Zingales M. A generalized model of elastic foundation based on long-range interactions: integral and fractional model. International Journal of Solids and Structures 2009; 46:3124-3137.

45. Failla G, Santini A, Zingales M. Solution strategies for 1d elastic continuum with long-range cohesive interactions: smooth and fractional decay. Mechanics Research Communications 2010; 37:13-21. 